\title{
DESENVOLVIMENTO DE TERMINOLOGIA DESCRITIVA PARA WARMED-OVER FLAVOR EM CARNE ASSADA BOVINA
}

\author{
Moacir Evandro Lage ${ }^{1}$, Helena TeiXeira Godoy ${ }^{2}$, Helena Maria ANDré Bolini ${ }^{2}$, RaPHAEl \\ ROCHA DE OLIVEIRA ${ }^{3}$, ANTÔNIO NONATO DE OliveIRA ${ }^{1}$, EdMAR SOARES NiCOlaU ${ }^{1}$, Cíntia Silva \\ MINAFRA E REZENDE ${ }^{1}$
}

${ }^{1}$ Professores doutores da Universidade Federal de Goiás, Goiânia, GO, Brasil - moacirlage @ hotmail.com

${ }^{2}$ Professores doutores da Universidade Estadual de Campinas, Campinas, SP, Brasil

${ }^{3}$ Doutorando da Universidade Federal de Goiás, Goiânia, GO, Brasil

RESUMO

\begin{abstract}
Foi avaliado o desenvolvimento de warmed-over flavor (WOF) em carne assada bovina através da análise sensorial descritiva utilizando-se os músculos longo dorsal, semitendinoso e supra-espinhoso de novilhos da raça Nelore. Houve uma pré-seleção para recrutamento dos provadores, de 45 indivíduos inscritos, 35 foram pré-selecionados, destes, 24 foram avaliados com testes de diferença (quatro testes triangulares) para determinar a habilidade em discriminar diferenças de WOF em amostras de carne assada. Após os testes, 13 indivíduos foram selecionados para determinar o Perfil Sensorial de cada amostra. Inicialmente, 70 termos foram propostos pela equipe
\end{abstract}

sensorial para a análise descritiva quantitativa, mas foram eliminados os termos que não apresentaram relevância para o produto, que não contribuíram para a discriminação de WOF na carne assada e os que apresentaram dificuldade de ser detectados pelos provadores. Restaram ao final 18 termos, sendo todos utilizados nos três tipos de músculos analisados. A análise descritiva quantitativa pode ser realizada para a obtenção de resultados satisfatórios para a pesquisa de warmed-over flavor em carne assada em experimentos científicos e para o desenvolvimento de produtos.

PALAVRAS-CHAVE: análise sensorial, oxidação lipídica, sabor desagradável

\section{DEVELOPMENT OF DESCRIPTIVE TERMINOLOGY FOR WARMED-OVER FLAVOR IN BOVINE ROAST-BEEF}

\section{ABSTRACT}

We evaluated the warmed-over flavor development (WOF) in bovine roast-beef through adescriptive sensory analysis using the Longissimus dorsi, Semitendinosus and Supraspinatus muscles of Nellore steers. There was a preselection for recruitment of assessors. Forty-five individuals were registered, being 35 preselected, from these, 24 were evaluated by difference tests (triangular tests) to determine the ability to discriminate WOF's differences in roast-beef samples. After the tests, 13 individuals were selected to determine the sensory profile of each sample. Initially, the sensory team proposed 70 terms for the quantitative descriptive analysis, but terms that were not relevant for the product, or did not contribute for WOF's discrimination in roast-beef as well as the ones that were not easily differentiated by the selected assessors were eliminated. The remained 18 terms were used in the three kinds of analyzed muscles. We concluded that quantitative descriptive analysis can be accomplished to obtain satisfactory results for warmed-over flavor research in roast-beef in both scientific experiments and product development.

KEYWORDS: lipid oxidation, off-flavor, sensory analysis 


\section{INTRODUÇÃO}

A oxidação lipídica é uma das maiores causas de deterioração na qualidade da carne e produtos cárneos. O desenvolvimento da rancidez oxidativa em produtos cárneos começa logo após a morte e continua a aumentar em intensidade até os produtos tornarem-se inaceitáveis para o consumidor ${ }^{1}$. O sabor torna-se uma propriedade crítica de qualquer produto alimentar quando difere daquele esperado pelo consumidor; em outras palavras, os consumidores não aceitarão sabores desagradáveis nos produtos cárneos pré-cozidos. $\mathrm{O}$ rápido desenvolvimento de "sabores desagradáveis" nesses produtos é um grande desafio aos processadores de carne devido ao aumento da demanda por carnes pré-cozidas nas últimas décadas ${ }^{2}$.

O termo "warmed-over flavor" (sabor de requentado - WOF) foi utilizado primeiramente por Tims e Watts ${ }^{3}$, que o definiram como o rápido início da rancidez em carnes cozidas durante a estocagem sob temperatura de refrigeração. Produtos bovinos pré-cozidos e reaquecidos após um curto período de estocagem sob refrigeração desenvolvem esse sabor desagradável distinto referido como WOF, que é considerado um problema de qualidade no setor de alimentos, em que o reaquecimento de carnes antes de servir é largamente utilizado. Muitos relatos indicam que o WOF está associado com a autoxidação dos ácidos graxos polinsaturados, principalmente nos fosfolipídios, e que o ferro, em várias formas, é um catalizador importante nas reações ${ }^{1,4}$.

Há vários estudos investigando como o cozimento, incluindo fatores como cozimento, temperatura, tempo e temperatura interna final ${ }^{5,6}$, pode influenciar o desenvolvimento de WOF. Os efeitos de tais parâmetros de cozimento são relacionados às diferenças na formação de produtos da reação de Maillard (PRM) na carne, que podem ser antioxidantes apropriados para prevenção efetiva do desenvolvimento de WOF em carnes cozidas ${ }^{1}$.

Algumas pesquisas mostram que a análise sensorial descritiva é uma ótima ferramenta para a avaliação de WOF em carnes $^{7-10}$. Este estudo foi desenvolvido com o objetivo de desenvolver uma terminologia descritiva para WOF em carne assada bovina.

\section{MATERIAL E MÉTODOS}

Os músculos longo dorsal (LL; contra-filé), semi-tendinoso (ST; lagarto) e supra-espinhoso (SS; peixinho) maturados e congelados, de novilhos da raça Nelore, foram mantidos a $5{ }^{\circ} \mathrm{C}$ por aproximadamente 12 horas para descongelar, permitindo, assim, o corte em bifes com $1,5 \mathrm{~cm}$ de espessura. Esses músculos foram escolhidos por apresentarem diferentes características, como conteúdo de mioglobina, de lipídios e tecido conjuntivo, além de outras. Os bifes foram assados em forno à temperatura de $150{ }^{\circ} \mathrm{C}$ (os bifes de ST foram assados envolvidos em papel alumínio) até atingirem a temperatura interna de $70{ }^{\circ} \mathrm{C}$ e, após as bordas serem retiradas, foram cortados em cubos de $\pm 1 \mathrm{~cm}^{3}$, evitando-se tecido conjuntivo e gorduroso visível. Os cubos de carne assada foram colocados juntos e misturados para se aleatorizar a amostragem, então, em número de três, foram cobertos com papel manteiga, embalados com sacos de polietileno $(20 \times 30 \mathrm{~cm})$ e mantidos sob refrigeração $\left( \pm 5^{\circ} \mathrm{C}\right)$ até sua utilização $(1,2$, e 3 dias), quando foram reaquecidos em banho-maria a $70{ }^{\circ} \mathrm{C}$ por 5 a 10 minutos e servidos aos provadores.

Houve uma pré-seleção para recrutamento dos provadores. Quarenta e cinco indivíduos inscritos preencheram as fichas de recrutamento, destes, foram pré-selecionados 35, que estavam dentro da faixa etária de 20-50 anos. Esse número foi necessário, pois os provadores eram inexperientes e era desejado, ao fim do treinamento, um número de 10 a 15 provadores. Os indivíduos recrutados eram estudantes ou funcionários da FEA-UNICAMP, Campinas-SP, Brasil. O contato foi feito por meio da Ficha de Recrutamento com informações sobre os testes e solicitação de informações sobre os interessados como: disponibilidade de tempo, saúde, conhecimento e interesse no produto, dados pessoais, entre outros. Dos 35 indivíduos pré-selecionados através da Ficha de Recrutamento, 24 foram avaliados com testes de diferença para determinar a habilidade em discriminar diferenças de WOF em amostras de carne assada. Os 11 pré-selecionados restantes não compareceram a todas avaliações. Foi utilizado o teste triangular, devido à probabilidade de se acertar ao acaso um terço, com isso obtinha-se maior eficiência estatística do que os testes de diferença simples (probabilidade de $1 / 2$ ). O grau de dificuldade dos testes foi ajustado utilizando-se amostras dos diferentes tratamentos, que foram 1,2 e 3 dias sob refrigeração após o cozimento.

Foram feitos quatro testes triangulares: o primeiro comparou carne recém preparada com carne reaquecida após 1 dia; o segundo comparou 
carne assada recém preparada e carne assada reaquecida após 3 dias de conservação sob refrigeração; o terceiro comparou carne reaquecida após 1 dia de conservação sob refrigeração com carne reaquecida após 2 dias; o quarto teste comparou carne assada reaquecida após 1 dia de conservação com carne reaquecida após 3 dias.

Todos os testes sensoriais foram realizados no Laboratório de Análise Sensorial do DEPAN da Faculdade de Engenharia de Alimentos (FEA UNICAMP), em cabines individuais, com utilização de iluminação branca para avaliação da aparência e com iluminação vermelha para avaliação do aroma, sabor e textura. Para a obtenção da lista de descritores e treinamento dos provadores, utilizou-se uma mesa redonda onde foram realizadas as discussões abertas, sob a coordenação de um indivíduo com conhecimento teórico e prático sobre o produto.

$\mathrm{O}$ desenvolvimento da terminologia descritiva das amostras de carne assada (LL, ST e SS) foi conduzido utilizando-se o Método de Rede $^{13}$, em que são descritas, pelos provadores, as similaridades e diferenças entre um par de amostras, criando termos descritivos. Os produtos foram apresentados aos provadores, em cabines individuais de avaliação sensorial. Os componentes da equipe foram solicitados a avaliar as amostras utilizando uma ficha para desenvolvimento da terminologia descritiva.

Após cada provador ter gerado seus próprios termos para descrever as similaridades e as diferenças entre as amostras, discussões em grupo foram conduzidas sob a supervisão do líder com o objetivo de agrupar termos descritivos semelhantes e gerar amostras referências.

Durante as sessões de treinamento, os provadores foram solicitados a avaliar a intensidade de cada atributo nas diferentes amostras utilizando a ficha de avaliação previamente desenvolvida pela equipe sensorial.

Para a avaliação final dos provadores, foi feita a análise descritiva das amostras de carne assada em três repetições utilizando-se uma Ficha de Avaliação Descritiva produzida com cada atributo empregando-se uma escala não estruturada de $9 \mathrm{~cm}$ ancorada em seus extremos com as palavras pouco ou nenhum e muito indicando a intensidade. Cada provador recebeu três amostras de cada período de refrigeração (1, 2 e 3 dias) para avaliação, devidamente casualizadas. $\mathrm{O}$ procedimento foi repetido para cada tipo de músculo.

O perfil sensorial das três amostras de carne assada (contra-filé, lagarto e peixinho) foi determinado pelos provadores da equipe sensorial descritiva, treinados e selecionados conforme descrito anteriormente. Utilizou-se um delineamento experimental de blocos completos casualizados ${ }^{12}$. Os resultados das avaliações feitas por estes provadores foram submetidos à Análise de Variância através do programa estatístico SAS ${ }^{11}$.

A análise de variância foi executada para os resultados de cada provador (Fontes de Variação: amostras, repetições). Provadores que apresentaram bom poder discriminativo ( $\mathrm{p}($ amostra $) \leq 0,50)$, boa reprodutibilidade nos julgamentos (p(repetições) $>$ $0,05)$ e consenso com os demais membros do grupo, foram selecionados para compor a equipe descritiva treinada. Das análises de variância realizadas, observou-se também os valores de $\mathrm{F}$ provador, $\mathrm{F}$ amostra e $\mathrm{F}$ interação provador $\mathrm{x}$ amostra, dos quais, respectivamente, as significâncias de $p>0,05$, $\mathrm{p}<0,05$ e $\mathrm{p}>0,05$ foram consideradas ideais. A análise estatística dos dados foi executada a partir dos valores obtidos por triplicata.

\section{RESULTADOS E DISCUSSÃO}

Dos 24 indivíduos testados, 13 foram selecionados após os quatro testes triangulares que indicaram percepção de WOF (mais de $70 \%$ de acertos, ou acerto em pelo menos três testes). $\mathrm{O}$ primeiro (0 dia X 1 dia), o segundo (0 dia $X 3$ dias) e o quarto (1 dia $X 3$ dias) testes triangulares mostraram diferença significativa $(\mathrm{P}<0,05)$ entre as amostras analisadas comparando-se o número de acertos $(18 / 24,16 / 24$ e 16/24) com a tabela para teste triangular ${ }^{13}$, enquanto no terceiro teste 1 dia $\mathrm{X}$ 2 dias $(12 / 24)$ só havia diferença com significância de $10 \%$. O Perfil Sensorial de cada amostra foi determinado por estes provadores segundo a metodologia de análise descritiva quantitativa ${ }^{12,13}$, modificada neste estudo pela exclusão de atributos de aparência e textura.

Alguns termos e referências foram baseados ou dirigidos para aqueles descritos na literatura ${ }^{7,10}$. Sessões suplementares de avaliação das amostras, das referências e de discussão em grupo resultaram no uso consensual de termos descritivos pela equipe sensorial. Para maior compreensão e consenso dos provadores, foram estabelecidas definições de cada termo descritivo pela equipe sensorial (Quadro 1). 
Quadro 1. Definição dos termos descritivos e referências usadas como extremos de escala de intensidade na ADQ de carne assada para os atributos de aroma, gosto e sabor

\begin{tabular}{|c|c|c|c|c|}
\hline ASPECTO & TERMO & DEFINIÇÃO & REFERÊNCIA MÍNIMA & $\begin{array}{l}\text { REFERÊNCIA } \\
\text { MÁXIMA }\end{array}$ \\
\hline \multirow[t]{8}{*}{ Aroma } & Carne assada & $\begin{array}{l}\text { Intensidade do aroma } \\
\text { associado à carne assada. }\end{array}$ & $\begin{array}{l}\text { Carne mal-passada - carne assada } \\
\text { em forno a } 150^{\circ} \mathrm{C} \text { até a } \\
\text { temperatura interna de } 60^{\circ} \mathrm{C} \\
\text { (relacionado pelo grupo ao gosto } \\
\text { de carne crua e gosto de sangue) }\end{array}$ & $\begin{array}{l}\text { Carne bem passada - } \\
\text { carne assada em forno a } \\
150^{\circ} \mathrm{C} \text { até a temperatura } \\
\text { interna de } 80^{\circ} \mathrm{C}\end{array}$ \\
\hline & $\begin{array}{l}\text { Carne de } \\
\text { frango }\end{array}$ & $\begin{array}{l}\text { Intensidade do aroma } \\
\text { associado ao coração de } \\
\text { frango cozido. }\end{array}$ & Carne mal-passada & $\begin{array}{l}\text { Coração de frango cozido } \\
\text { em água a } 98^{\circ} \mathrm{C} / 40 \mathrm{~min} .\end{array}$ \\
\hline & $\begin{array}{l}\text { Óleo de } \\
\text { linhaça }\end{array}$ & $\begin{array}{l}\text { Intensidade do aroma } \\
\text { associado ao óleo de linhaça } \\
\text { (relacionado pelo grupo ao } \\
\text { aroma de mato, chá, passas e } \\
\text { ração) }\end{array}$ & Água filtrada & Òleo de linhaça \\
\hline & Peixe & $\begin{array}{l}\text { Intensidade do aroma } \\
\text { associado ao óleo de fígado de } \\
\text { bacalhau }\end{array}$ & Água filtrada & $\begin{array}{l}\text { Óleo de fígado de } \\
\text { bacalhau }\end{array}$ \\
\hline & Noz & $\begin{array}{l}\text { Intensidade do aroma } \\
\text { associado a noz }\end{array}$ & Água filtrada & Noz \\
\hline & Papelão & $\begin{array}{l}\text { Intensidade do aroma } \\
\text { associado ao papelão }\end{array}$ & Água filtrada & Tiras de papelão molhado \\
\hline & $\begin{array}{l}\text { Óleo vegetal } \\
\text { oxidado }\end{array}$ & $\begin{array}{l}\text { Intensidade do aroma } \\
\text { associado ao óleo de soja } \\
\text { oxidado (relacionado pelo } \\
\text { grupo ao aroma de pastelaria) }\end{array}$ & Água filtrada & $\begin{array}{l}\text { Óleo de soja aquecido a } \\
235^{\circ} \mathrm{C} / 40 \mathrm{~min} \text {. }\end{array}$ \\
\hline & Ovo cozido & $\begin{array}{l}\text { Intensidade do aroma } \\
\text { associado ao ovo cozido. }\end{array}$ & Carne mal-passada & $\begin{array}{l}\text { Ovo cozido em água a } \\
98^{\circ} \mathrm{C} / 20 \mathrm{~min} .\end{array}$ \\
\hline \multirow[t]{5}{*}{ Gosto } & Doce & $\begin{array}{l}\text { Intensidade do gosto associado } \\
\text { à sacarose. }\end{array}$ & Água filtrada & $\begin{array}{l}\text { Solução de sacarose a } \\
1 \% \text {. }\end{array}$ \\
\hline & Salgado & $\begin{array}{l}\text { Intensidade do gosto associado } \\
\text { ao cloreto de sódio. }\end{array}$ & Água filtrada & Solução $0,5 \%$ de $\mathrm{NaCl}$. \\
\hline & Ácido & $\begin{array}{l}\text { Intensidade do gosto associado } \\
\text { ao ác. cítrico. }\end{array}$ & Água filtrada & $\begin{array}{l}\text { Solução 0,03\% de ác. } \\
\text { cítrico. }\end{array}$ \\
\hline & Amargo & $\begin{array}{l}\text { Intensidade do gosto associado } \\
\text { à cafeína. }\end{array}$ & Água filtrada & $\begin{array}{l}\text { Solução } 0,03 \% \text { de } \\
\text { cafeína. }\end{array}$ \\
\hline & Umami & $\begin{array}{l}\text { Intensidade do gosto associado } \\
\text { ao glutamato monossódico. }\end{array}$ & Água filtrada & $\begin{array}{l}\text { Solução de glutamato } \\
\text { monossódico } 0,5 \% \text {. }\end{array}$ \\
\hline \multirow[t]{4}{*}{ Sabor } & Carne assada & $\begin{array}{l}\text { Intensidade do sabor associado } \\
\text { à carne assada. }\end{array}$ & Carne mal-passada & Carne bem passada \\
\hline & $\begin{array}{l}\text { Carne de } \\
\text { frango }\end{array}$ & $\begin{array}{l}\text { Intensidade do sabor associado } \\
\text { ao coração de frango cozido. }\end{array}$ & Carne mal-passada & $\begin{array}{l}\text { Coração de frango cozido } \\
\text { em água a } 98^{\circ} \mathrm{C} / 40 \mathrm{~min} .\end{array}$ \\
\hline & $\begin{array}{l}\text { Óleo vegetal } \\
\text { oxidado }\end{array}$ & $\begin{array}{l}\text { Intensidade do sabor associado } \\
\text { ao óleo de soja oxidado }\end{array}$ & Água filtrada & $\begin{array}{l}\text { Óleo de soja aquecido a } \\
235^{\circ} \mathrm{C} / 40 \mathrm{~min} \text {. }\end{array}$ \\
\hline & $\mathrm{Noz}$ & $\begin{array}{l}\text { Intensidade do sabor associado } \\
\text { a noz }\end{array}$ & Água filtrada & Noz \\
\hline $\begin{array}{l}\text { "After } \\
\text { taste" }\end{array}$ & $\begin{array}{l}\text { "After taste } \\
\text { umami" }\end{array}$ & $\begin{array}{l}\text { Gosto umami residual que fica } \\
\text { após a mastigação }\end{array}$ & Água filtrada & $\begin{array}{l}\text { Solução de glutamato } \\
\text { monossódico } 0,5 \% \text {. }\end{array}$ \\
\hline
\end{tabular}

De 70 termos inicialmente propostos reduziu-se para 29. Os critérios para a escolha desses termos foi, em primeira instância, o número de indicações dos provadores para cada termo, a relevância para o produto, o poder de discriminação entre as amostras, a ausência de redundância e a percepção clara pelo grupo de provadores. Por fim, termos que não se enquadravam nessas premissas foram eliminados. Dentre os 29 termos selecionados, aqueles relativos à aparência e à textura, ainda que importantes na caracterização da amostra, foram eliminados, já que não contribuíam para a discriminação de WOF na carne assada, restando, assim, 23 termos. Desses 23 termos, cinco (aroma de carne cozida, aroma de charque, aroma de castanha do pará, 
sabor de carne cozida e sabor de charque) foram removidos devido à dificuldade de alguns componentes do grupo em detectá-los, restando ao final 18 termos (Quadro 1). Além disso, o atributo sabor de óleo vegetal, pelos mesmos motivos citados, foi substituído por sabor de óleo vegetal oxidado, sendo que este último se mostrou muito importante na diferenciação das amostras. Os termos propostos para os três tipos de músculo analisados (LL, ST e SS) foram os mesmos, descartando-se a necessidade de uma ficha de análise descritiva específica para cada um.

Por ser a carne assada um produto complexo, $\mathrm{e}$ os provadores inexperientes, foram necessárias 20 sessões, com duas horas cada, para o desenvolvimento da terminologia e treinamento dos provadores. Entre essas sessões, estão as sessões de "retreinamento", devido à ausência da maioria dos provadores em certos períodos (ex: férias), e de "reavaliações", devido à dificuldade de alguns provadores na avaliação de alguns atributos.

Em cada sessão, foram colocados à disposição dos provadores os materiais de referência e a definição de cada termo descritivo. As sessões de treinamento enfocavam prioritariamente os atributos com maior grau de dificuldade para os provadores, procurando sempre se chegar ao consenso entre eles.

O desempenho de cada provador foi avaliado. Todos os provadores apresentaram boa capacidade de discriminação dos atributos avaliados, repetibilidade (Tabelas 1a, 1b e 1c) e consenso entre a equipe na maioria dos atributos. Observaram-se poucos valores indesejáveis para discriminação das amostras e repetibilidade, sendo estes distribuídos pelos provadores, atributos e tipo de amostra de carne assada (LL, ST e SS).

Tabela 1a-Valores de p repetição (valores acima) e p amostra (valores abaixo) obtidos por nove provadores em cada atributo para seleção da equipe (valores desejáveis $\mathrm{p}$ amostra $<0,50$ e p repetição $\geq 0,05$ ) para avaliação de contra-filé (LL) assado

\begin{tabular}{|c|c|c|c|c|c|c|c|c|c|}
\hline Atributo & P1 & $\mathbf{P 2}$ & P3 & $\mathbf{P 4}$ & P5 & P6 & P7 & P8 & P9 \\
\hline \multirow{2}{*}{ Aroma Carne Assada } & 0,0037 & 0,0310 & 0,0001 & 0,0007 & 0,3469 & 0,0001 & 0,0001 & 0,0001 & 0,0020 \\
\hline & 0,5879 & 0,1854 & 0,5227 & 0,5003 & 0,5775 & 0,2684 & 0,2352 & 0,8763 & 0,3719 \\
\hline \multirow{2}{*}{ Aroma Carne Frango } & 0,1311 & 0,1813 & 0,0007 & 0,2298 & 0,0152 & 0,0002 & 0,0001 & 0,3336 & 0,0149 \\
\hline & 0,5834 & 0,9000 & 0,9468 & $\mathbf{0 , 0 2 3 8}$ & 0,2512 & 0,5606 & 0,3876 & 0,0672 & 0,9913 \\
\hline \multirow{2}{*}{ Aroma Peixe } & 0,0422 & 0,3031 & 0,4187 & 0,0152 & 0,0648 & 0,0305 & 0,3768 & 0,0084 & 0,0683 \\
\hline & 0,9942 & 0,7443 & 0,4506 & 0,1755 & 0,9691 & 0,3085 & 0,8312 & 0,9044 & 0,2249 \\
\hline \multirow{2}{*}{ Aroma Óleo Linhaça } & 0,0172 & 0,6774 & 0,0522 & 0,0015 & 0,0013 & 0,0001 & 0,4432 & 0,6524 & 0,0001 \\
\hline & 0,8747 & 0,1705 & 0,8212 & 0,8782 & 0,2420 & 0,0831 & 0,7982 & 0,0206 & 0,8881 \\
\hline \multirow{2}{*}{ Aroma Óleo Vegetal Oxidado } & 0,0181 & 0,1886 & 0,1417 & 0,0036 & 0,5432 & 0,4869 & 0,9588 & 0,5144 & 0,0970 \\
\hline & 0,7873 & 0,7302 & 0,4522 & 0,4071 & 0,2375 & 0,6509 & 0,9889 & 0,3124 & 0,5821 \\
\hline \multirow{2}{*}{ Aroma Noz } & 0,1104 & 0,4243 & 0,0523 & 0,2786 & 0,1427 & 0,0003 & 0,1601 & 0,0290 & $\mathbf{0 , 8 8 3 8}$ \\
\hline & 0,7199 & 0,0941 & 0,5881 & 0,6147 & 0,8308 & 0,2123 & 0,0462 & 0,2623 & 0,4207 \\
\hline \multirow{2}{*}{ Aroma Ovo Cozido } & $\mathbf{0 , 7 7 5 4}$ & 0,0198 & 0,2898 & 0,2738 & 0,3515 & 0,4651 & 0,1330 & 0,0002 & 0,0401 \\
\hline & 0,7469 & 0,7864 & 0,4179 & 0,1403 & 0,4790 & 0,3609 & 0,3233 & 0,8981 & 0,1216 \\
\hline \multirow{2}{*}{ Aroma Papelão } & 0,6657 & $\mathbf{0 , 9 3 2 8}$ & 0,1163 & $\mathbf{0 , 5 6 2 7}$ & 0,0089 & 0,0535 & 0,9054 & 0,0408 & 0,0005 \\
\hline & 0,7498 & 0,1926 & 0,2710 & 0,4342 & 0,4554 & 0,6097 & 0,7060 & 0,9573 & 0,2468 \\
\hline \multirow{2}{*}{ Gosto Salgado } & 0,2469 & 0,1404 & 0,0643 & $\mathbf{0 , 5 3 2 4}$ & 0,2148 & 0,2380 & 0,3462 & 0,1300 & 0,3759 \\
\hline & 0,8123 & 0,3220 & 0,3830 & 0,0478 & 0,3880 & 0,5580 & 0,9564 & 0,2859 & 0,7592 \\
\hline \multirow{2}{*}{ Gosto Doce } & 0,0120 & 0,0001 & 0,3425 & 0,2602 & 0,1347 & 0,2836 & 0,0002 & 0,4187 & 0,0001 \\
\hline & 0,8126 & 0,0603 & 0,4357 & 0,8064 & 0,7662 & 0,3475 & 0,3585 & 0,7450 & 0,2820 \\
\hline \multirow{2}{*}{ Gosto Ácido } & 0,0005 & 0,0054 & 0,0031 & 0,2985 & $\mathbf{0 , 8 3 1 7}$ & 0,0324 & 0,1773 & 0,0019 & 0,3060 \\
\hline & 0,2373 & 0,9827 & 0,0751 & 0,6354 & 0,6132 & 0,3882 & 0,9188 & 0,1092 & 0,6257 \\
\hline \multirow{2}{*}{ Gosto Amargo } & 0,0006 & 0,0007 & 0,0001 & 0,0745 & 0,4870 & 0,9325 & 0,0002 & 0,1916 & 0,1604 \\
\hline & 0,4485 & 0,4142 & 0,4031 & 0,6361 & 0,6447 & 0,7594 & 0,5101 & 0,2984 & 0,6862 \\
\hline \multirow{2}{*}{ Gosto Umami } & 0,0546 & 0,0025 & 0,1743 & 0,0751 & 0,0980 & 0,0034 & 0,7434 & 0,2507 & 0,0135 \\
\hline & 0,8136 & 0,1579 & 0,2812 & 0,1368 & 0,9104 & 0,4653 & 0,5973 & 0,7419 & 0,0627 \\
\hline \multirow{2}{*}{ Sabor Carne Assada } & 0,6817 & 0,0001 & 0,0001 & 0,0001 & 0,4312 & 0,0002 & 0,0004 & 0,0002 & 0,0001 \\
\hline & 0,6599 & 0,3387 & 0,6298 & 0,5288 & 0,1527 & 0,8709 & 0,6150 & 0,3756 & 0,5537 \\
\hline \multirow{2}{*}{ Sabor Carne Frango } & 0,0010 & 0,0937 & 0,0001 & 0,1758 & 0,5936 & 0,6451 & 0,0001 & 0,0678 & 0,0052 \\
\hline & 0,5849 & 0,4569 & 0,3701 & 0,0278 & 0,1844 & 0,2019 & 0,1877 & 0,6082 & 0,4466 \\
\hline \multirow{2}{*}{ Sabor Óleo Vegetal Oxidado } & 0,0220 & $\mathbf{0 , 5 8 6 3}$ & 0,2724 & 0,8884 & 0,2365 & 0,0002 & 0,1233 & 0,6154 & 0,0233 \\
\hline & 0,9160 & 0,7503 & 0,4813 & 0,7612 & 0,6342 & 0,3232 & 0,3130 & 0,5454 & 0,7884 \\
\hline \multirow{2}{*}{ Sabor Noz } & 0,1254 & 0,4664 & 0,7149 & 0,1691 & 0,2450 & 0,2493 & 0,8809 & 0,0010 & 0,4203 \\
\hline & 0,5172 & 0,5484 & 0,5620 & 0,6891 & 0,8971 & 0,6865 & 0,8022 & 0,4057 & 0,2549 \\
\hline \multirow{2}{*}{ Aftertaste Umami } & 0,1327 & 0,0664 & 0,0169 & 0,0005 & 0,0015 & 0,0027 & 0,6913 & 0,0377 & 0,0550 \\
\hline & 0,6611 & 0,6315 & 0,6487 & 0,1799 & 0,1895 & 0,0777 & 0,6151 & 0,6320 & 0,1416 \\
\hline
\end{tabular}

Os valores indesejáveis estão marcados em negrito.

Nas tabelas 1a, 1b e 1c é mostrado o número de valores indesejáveis para repetibilidade $(\mathrm{p}<0,05)$ e 
para discriminação de amostras $(p>0,50)$ em cada atributo analisado por nove provadores no caso dos músculos LL e ST e por oito provadores no caso do músculo SS (um dos provadores não pôde realizar as análises). Pode-se observar que houve um pequeno número de valores indesejáveis. Foram encontradas mais inconsistências para discriminação de amostras, sendo os atributos relacionados ao óleo vegetal oxidado (sabor e aroma) que apresentaram maior volume de ocorrências.

Tabela $1 \mathrm{~b}$-Valores de p repetição (valores acima) e p amostra (valores abaixo) obtidos por nove provadores em cada atributo para seleção da equipe (valores desejáveis $\mathrm{p}$ amostra $<0,50$ e p repetição $\geq 0,05$ ) para avaliação de lagarto (ST) assado

\begin{tabular}{|c|c|c|c|c|c|c|c|c|c|}
\hline Atributo & P1 & $\mathbf{P 2}$ & $\mathbf{P 3}$ & P4 & P5 & P6 & P7 & P8 & P9 \\
\hline AROMA CARNE ASSADA & $\begin{array}{l}0,0443 \\
0,8078\end{array}$ & $\begin{array}{l}0,0053 \\
0,2259\end{array}$ & $\begin{array}{l}0,0001 \\
0,2858\end{array}$ & $\begin{array}{l}0,0025 \\
0,6391\end{array}$ & $\begin{array}{l}0,0544 \\
0,2008\end{array}$ & $\begin{array}{l}0,3483 \\
0,6553\end{array}$ & $\begin{array}{l}0,0469 \\
0,2338\end{array}$ & $\begin{array}{l}0,0001 \\
0,5448\end{array}$ & $\begin{array}{l}0,1993 \\
0,3140\end{array}$ \\
\hline AROMA CARNE FRANGO & $\begin{array}{l}0,0165 \\
0,2635\end{array}$ & $\begin{array}{l}0,0006 \\
0,3972\end{array}$ & $\begin{array}{l}0,0001 \\
0,2594\end{array}$ & $\begin{array}{l}0,0001 \\
0,3016\end{array}$ & $\begin{array}{l}0,2234 \\
0,2239\end{array}$ & $\begin{array}{l}0,1189 \\
0,0961\end{array}$ & $\begin{array}{l}0,0001 \\
0,6632\end{array}$ & $\begin{array}{l}0,0004 \\
0,2398\end{array}$ & $\begin{array}{l}\mathbf{0 , 5 2 7 3} \\
0,7983\end{array}$ \\
\hline AROMA PEIXE & $\begin{array}{l}0,0007 \\
\mathbf{0 , 0 1 0 5}\end{array}$ & $\begin{array}{l}0,0440 \\
0,4098\end{array}$ & $\begin{array}{l}0,0001 \\
0,3945\end{array}$ & $\begin{array}{l}0,0001 \\
0,0864\end{array}$ & $\begin{array}{l}0,0284 \\
0,6830\end{array}$ & $\begin{array}{l}0,0001 \\
0,4198\end{array}$ & $\begin{array}{l}0,0001 \\
0,9361\end{array}$ & $\begin{array}{l}0,0046 \\
0,3968\end{array}$ & $\begin{array}{l}0,0001 \\
\mathbf{0 , 0 0 0 5}\end{array}$ \\
\hline AROMA ÓLEO LINHAÇA & $\begin{array}{l}0,0005 \\
0,4977\end{array}$ & $\begin{array}{l}0,0846 \\
0,2571\end{array}$ & $\begin{array}{l}0,0001 \\
\mathbf{0 , 0 3 1 4}\end{array}$ & $\begin{array}{l}0,1555 \\
0,6753\end{array}$ & $\begin{array}{l}0,0830 \\
0,4576\end{array}$ & $\begin{array}{l}0,2486 \\
0,5242\end{array}$ & $\begin{array}{l}0,0021 \\
0,1239\end{array}$ & $\begin{array}{l}0,0008 \\
0,3251\end{array}$ & $\begin{array}{l}0,0180 \\
0,1676\end{array}$ \\
\hline $\begin{array}{l}\text { AROMA ÓLEO VEGETAL } \\
\text { OXIDADO }\end{array}$ & $\begin{array}{l}0,0001 \\
\mathbf{0 , 0 2 9 4}\end{array}$ & $\begin{array}{l}\mathbf{0 , 5 0 1 4} \\
0,4211\end{array}$ & $\begin{array}{l}0,0148 \\
0,3523\end{array}$ & $\begin{array}{l}0,0051 \\
0,0859\end{array}$ & $\begin{array}{l}0,1944 \\
0,2881\end{array}$ & $\begin{array}{l}\mathbf{0 , 8 5 5 4} \\
0,2742\end{array}$ & $\begin{array}{l}0,0704 \\
0,3644\end{array}$ & $\begin{array}{l}0,0013 \\
0,5109\end{array}$ & $\begin{array}{l}0,2397 \\
0,5821\end{array}$ \\
\hline AROMA NOZ & $\begin{array}{l}0,0001 \\
\mathbf{0 , 0 0 9 2}\end{array}$ & $\begin{array}{l}0,0307 \\
0,7491\end{array}$ & $\begin{array}{l}0,3903 \\
0,9483\end{array}$ & $\begin{array}{l}0,0036 \\
0,2029\end{array}$ & $\begin{array}{l}\mathbf{0 , 8 6 4 1} \\
0,3830\end{array}$ & $\begin{array}{l}0,1909 \\
0,3689\end{array}$ & $\begin{array}{l}\mathbf{0 , 9 0 5 5} \\
0,2392\end{array}$ & $\begin{array}{l}\mathbf{0 , 6 7 8 8} \\
0,7287\end{array}$ & $\begin{array}{l}0,3111 \\
0,4442\end{array}$ \\
\hline AROMA OVO COZIDO & $\begin{array}{l}0,0001 \\
0,7094\end{array}$ & $\begin{array}{l}0,0001 \\
0,4719\end{array}$ & $\begin{array}{l}0,0001 \\
0,2690\end{array}$ & $\begin{array}{l}0,0001 \\
0,5252\end{array}$ & $\begin{array}{l}0,3758 \\
0,6515\end{array}$ & $\begin{array}{l}0,0003 \\
0,6855\end{array}$ & $\begin{array}{l}0,0007 \\
0,4458\end{array}$ & $\begin{array}{l}0,0001 \\
0,0784\end{array}$ & $\begin{array}{l}0,0005 \\
\mathbf{0 , 0 1 5 1}\end{array}$ \\
\hline AROMA PAPELÃO & $\begin{array}{l}0,0220 \\
0,1758\end{array}$ & $\begin{array}{l}0,0001 \\
0,2458\end{array}$ & $\begin{array}{l}0,0031 \\
\mathbf{0 , 0 3 5 4}\end{array}$ & $\begin{array}{l}0,0023 \\
0,7482\end{array}$ & $\begin{array}{l}0,0017 \\
0,8465\end{array}$ & $\begin{array}{l}0,0903 \\
0,7330\end{array}$ & $\begin{array}{l}\mathbf{0 , 5 6 2 8} \\
0,0784\end{array}$ & $\begin{array}{l}0,4109 \\
0,6649\end{array}$ & $\begin{array}{l}0,3376 \\
0,4832\end{array}$ \\
\hline GOSTO SALGADO & $\begin{array}{l}0,3463 \\
0,9584\end{array}$ & $\begin{array}{l}0,1749 \\
0,6177\end{array}$ & $\begin{array}{l}0,2357 \\
0,8397\end{array}$ & $\begin{array}{l}0,0305 \\
0,1349\end{array}$ & $\begin{array}{l}0,1060 \\
0,5393\end{array}$ & $\begin{array}{l}\mathbf{0 , 6 5 3 1} \\
0,5700\end{array}$ & $\begin{array}{l}\mathbf{0 , 9 0 9 0} \\
0,5272\end{array}$ & $\begin{array}{l}0,0605 \\
0,5340\end{array}$ & $\begin{array}{l}0,0423 \\
0,5662\end{array}$ \\
\hline GOSTO DOCE & $\begin{array}{l}0,1374 \\
0,0862\end{array}$ & $\begin{array}{l}0,0032 \\
0,8034\end{array}$ & $\begin{array}{l}\mathbf{0 , 7 0 0 5} \\
0,3341\end{array}$ & $\begin{array}{l}\mathbf{0 , 5 6 9 5} \\
0,0951\end{array}$ & $\begin{array}{l}0,1446 \\
0,7886\end{array}$ & $\begin{array}{l}0,2671 \\
0,5804\end{array}$ & $\begin{array}{l}0,0001 \\
0,8860\end{array}$ & $\begin{array}{l}0,0004 \\
0,8361\end{array}$ & $\begin{array}{l}0,0071 \\
0,5370\end{array}$ \\
\hline GOSTO ÁCIDO & $\begin{array}{l}0,0001 \\
0,5589\end{array}$ & $\begin{array}{l}0,0001 \\
0,5436\end{array}$ & $\begin{array}{l}0,1385 \\
0,2933\end{array}$ & $\begin{array}{l}0,0245 \\
0,7590\end{array}$ & $\begin{array}{l}0,2528 \\
0,7538\end{array}$ & $\begin{array}{l}0,0332 \\
0,5617\end{array}$ & $\begin{array}{l}0,0001 \\
0,5482\end{array}$ & $\begin{array}{l}0,0001 \\
0,3131\end{array}$ & $\begin{array}{l}\mathbf{0 , 7 8 1 8} \\
0,8171\end{array}$ \\
\hline GOSTO AMARGO & $\begin{array}{l}0,0101 \\
0,7771\end{array}$ & $\begin{array}{l}0,0001 \\
0,1709\end{array}$ & $\begin{array}{l}0,0001 \\
0,1487\end{array}$ & $\begin{array}{l}\mathbf{0 , 8 0 0 4} \\
0,4365\end{array}$ & $\begin{array}{l}\mathbf{0 , 8 7 0 5} \\
0,0629\end{array}$ & $\begin{array}{l}0,0001 \\
0,2811\end{array}$ & $\begin{array}{l}0,0001 \\
0,3292\end{array}$ & $\begin{array}{l}0,0051 \\
0,3539\end{array}$ & $\begin{array}{l}0,5674 \\
0,1913\end{array}$ \\
\hline GOSTO UMAMI & $\begin{array}{l}0,0196 \\
0,5288\end{array}$ & $\begin{array}{l}0,0131 \\
0,9057\end{array}$ & $\begin{array}{l}0,0149 \\
0,1661\end{array}$ & $\begin{array}{l}0,0001 \\
0,1579\end{array}$ & $\begin{array}{l}0,1759 \\
0,4413\end{array}$ & $\begin{array}{l}0,1197 \\
0,8990\end{array}$ & $\begin{array}{l}0,0035 \\
0,6124\end{array}$ & $\begin{array}{l}\mathbf{0 , 6 0 0 5} \\
0,8929\end{array}$ & $\begin{array}{l}0,0443 \\
0,1879\end{array}$ \\
\hline SABOR CARNE ASSADA & $\begin{array}{l}0,1646 \\
0,9633\end{array}$ & $\begin{array}{l}0,0031 \\
0,9005\end{array}$ & $\begin{array}{l}0,0001 \\
0,4883\end{array}$ & $\begin{array}{l}0,0030 \\
0,5870\end{array}$ & $\begin{array}{l}0,4934 \\
0,4745\end{array}$ & $\begin{array}{l}0,0080 \\
0,2326\end{array}$ & $\begin{array}{l}0,0641 \\
0,3176\end{array}$ & $\begin{array}{l}0,0002 \\
0,5868\end{array}$ & $\begin{array}{l}0,0748 \\
0,5229\end{array}$ \\
\hline SABOR CARNE FRANGO & $\begin{array}{l}0,1239 \\
0,7858\end{array}$ & $\begin{array}{l}0,0052 \\
0,2990\end{array}$ & $\begin{array}{l}0,0001 \\
0,8160\end{array}$ & $\begin{array}{l}0,0001 \\
0,9197\end{array}$ & $\begin{array}{l}0,0071 \\
0,9603\end{array}$ & $\begin{array}{l}0,0001 \\
0,0961\end{array}$ & $\begin{array}{l}0,0014 \\
0,9104\end{array}$ & $\begin{array}{l}0,0367 \\
0,6912\end{array}$ & $\begin{array}{l}\mathbf{0 , 8 6 9 3} \\
0,6811\end{array}$ \\
\hline $\begin{array}{l}\text { SABOR ÓLEO VEGETAL } \\
\text { OXIDADO }\end{array}$ & $\begin{array}{l}0,0062 \\
\mathbf{0 , 0 4 7 4}\end{array}$ & $\begin{array}{l}0,0504 \\
0,0372\end{array}$ & $\begin{array}{l}0,0036 \\
0,9449\end{array}$ & $\begin{array}{l}0,0001 \\
\mathbf{0 , 0 4 6 0}\end{array}$ & $\begin{array}{l}\mathbf{0 , 8 9 7 3} \\
0,1229\end{array}$ & $\begin{array}{l}0,4224 \\
0,5413\end{array}$ & $\begin{array}{l}0,1818 \\
0,4255\end{array}$ & $\begin{array}{l}0,0020 \\
\mathbf{0 , 0 2 1 9}\end{array}$ & $\begin{array}{l}0,2470 \\
0,2565\end{array}$ \\
\hline SABOR NOZ & $\begin{array}{l}0,0305 \\
0,2127\end{array}$ & $\begin{array}{l}\mathbf{0 , 8 7 5 6} \\
0,1867\end{array}$ & $\begin{array}{l}0,3161 \\
0,2283\end{array}$ & $\begin{array}{l}0,3252 \\
0,5700\end{array}$ & $\begin{array}{l}0,1336 \\
0,1770\end{array}$ & $\begin{array}{l}0,0516 \\
0,5014\end{array}$ & $\begin{array}{l}\mathbf{0 , 6 0 3 0} \\
0,5546\end{array}$ & $\begin{array}{l}0,4023 \\
0,5672\end{array}$ & $\begin{array}{l}\mathbf{0 , 6 5 7 9} \\
0,1553\end{array}$ \\
\hline AFTERTASTE UMAMI & $\begin{array}{l}0,1025 \\
0,6474\end{array}$ & $\begin{array}{l}0,0034 \\
0,9123\end{array}$ & $\begin{array}{l}0,1312 \\
0,2393\end{array}$ & $\begin{array}{l}0,0001 \\
0,1592\end{array}$ & $\begin{array}{l}0,0174 \\
0,2008\end{array}$ & $\begin{array}{l}\mathbf{0 , 6 8 2 6} \\
0,4551\end{array}$ & $\begin{array}{l}0,0025 \\
0,5503\end{array}$ & $\begin{array}{l}0,4756 \\
0,8349\end{array}$ & $\begin{array}{l}0,0360 \\
0,1767\end{array}$ \\
\hline
\end{tabular}

Os valores indesejáveis estão marcados em negrito.

Observa-se também que o número de valores indesejáveis, tanto para repetibilidade quanto para discriminação de amostras, apresentou tendência a decrescer do primeiro para o terceiro músculo.
Provavelmente isto se deva a uma maior experiência adquirida pelos provadores, já que os músculos foram analisados na ordem em que se encontram as tabelas (1a, 1b e 1c). 
Tabela 1c-Valores de p repetição (valores acima) e p amostra (valores abaixo) obtidos por 9 provadores em cada atributo para seleção da equipe (valores desejáveis $p$ amostra $<0,50$ e p repetição $\geq 0,05$ ) para avaliação de peixinho (SS) assado

\begin{tabular}{|c|c|c|c|c|c|c|c|c|}
\hline ATRIBUTO & P1 & P2 & P3 & P4 & P5 & P6 & P7 & P8 \\
\hline AROMA CARNE ASSADA & $\begin{array}{l}0,0130 \\
0,5954\end{array}$ & $\begin{array}{l}0,4795 \\
0,1106\end{array}$ & $\begin{array}{l}0,0491 \\
0,1391\end{array}$ & $\begin{array}{l}0,0132 \\
0,9439\end{array}$ & $\begin{array}{l}0,1424 \\
0,1600\end{array}$ & $\begin{array}{l}\mathbf{0 , 5 6 3 5} \\
0,4166\end{array}$ & $\begin{array}{l}0,0140 \\
0,2257\end{array}$ & $\begin{array}{l}0,0001 \\
0,1475\end{array}$ \\
\hline AROMA CARNE FRANGO & $\begin{array}{l}0,1395 \\
0,6775\end{array}$ & $\begin{array}{l}0,1569 \\
0,6188\end{array}$ & $\begin{array}{l}0,0243 \\
0,1362\end{array}$ & $\begin{array}{l}0,0220 \\
0,7783\end{array}$ & $\begin{array}{l}0,0986 \\
0,1351\end{array}$ & $\begin{array}{l}0,0013 \\
0,9404\end{array}$ & $\begin{array}{l}0,0045 \\
0,8702\end{array}$ & $\begin{array}{l}0,0572 \\
0,6972\end{array}$ \\
\hline AROMA PEIXE & $\begin{array}{l}0,0001 \\
0,2643\end{array}$ & $\begin{array}{l}0,4731 \\
0,8788\end{array}$ & $\begin{array}{l}0,0001 \\
0,4067\end{array}$ & $\begin{array}{l}0,0493 \\
0,0816\end{array}$ & $\begin{array}{l}0,1531 \\
0,3010\end{array}$ & $\begin{array}{l}0,0001 \\
0,9562\end{array}$ & $\begin{array}{l}0,0001 \\
\mathbf{0 , 0 1 6 0}\end{array}$ & $\begin{array}{l}0,0021 \\
0,8662\end{array}$ \\
\hline AROMA ÓLEO LINHAÇA & $\begin{array}{l}0,0005 \\
0,3252\end{array}$ & $\begin{array}{l}0,3086 \\
0,3170\end{array}$ & $\begin{array}{l}0,0014 \\
0,1283\end{array}$ & $\begin{array}{l}0,2725 \\
0,6212\end{array}$ & $\begin{array}{l}0,3040 \\
0,1183\end{array}$ & $\begin{array}{l}0,0061 \\
0,4151\end{array}$ & $\begin{array}{l}0,0531 \\
0,0995\end{array}$ & $\begin{array}{l}0,2466 \\
0,3991\end{array}$ \\
\hline $\begin{array}{l}\text { AROMA ÓLEO VEGETAL } \\
\text { OXIDADO }\end{array}$ & $\begin{array}{l}0,0001 \\
0,2122\end{array}$ & $\begin{array}{l}0,4027 \\
0,2734\end{array}$ & $\begin{array}{l}\mathbf{0 , 5 0 2 6} \\
0,7908\end{array}$ & $\begin{array}{l}0,0329 \\
0,1542\end{array}$ & $\begin{array}{l}0,0001 \\
0,9051\end{array}$ & $\begin{array}{l}0,0038 \\
0,3542\end{array}$ & $\begin{array}{l}0,0707 \\
0,2974\end{array}$ & $\begin{array}{l}0,0864 \\
0,2269\end{array}$ \\
\hline AROMA NOZ & $\begin{array}{l}0,0001 \\
0,1956\end{array}$ & $\begin{array}{l}0,3955 \\
0,3614\end{array}$ & $\begin{array}{l}0,0085 \\
0,5944\end{array}$ & $\begin{array}{l}0,0244 \\
0,1795\end{array}$ & $\begin{array}{l}\mathbf{0 , 6 2 9 9} \\
0,1866\end{array}$ & $\begin{array}{l}0,1029 \\
\mathbf{0 , 0 1 1 5}\end{array}$ & $\begin{array}{l}0,1825 \\
0,1438\end{array}$ & $\begin{array}{l}0,0497 \\
0,5370\end{array}$ \\
\hline AROMA OVO COZIDO & $\begin{array}{l}0,0001 \\
0,5877\end{array}$ & $\begin{array}{l}\mathbf{0 , 8 1 5 0} \\
0,8223\end{array}$ & $\begin{array}{l}0,0001 \\
0,0936\end{array}$ & $\begin{array}{l}0,0001 \\
0,0905\end{array}$ & $\begin{array}{l}\mathbf{0 , 9 4 8 7} \\
0,1565\end{array}$ & $\begin{array}{l}0,0001 \\
0,1903\end{array}$ & $\begin{array}{l}0,0421 \\
0,1631\end{array}$ & $\begin{array}{l}0,0316 \\
0,9748\end{array}$ \\
\hline AROMA PAPELÃO & $\begin{array}{l}0,0277 \\
0,1318\end{array}$ & $\begin{array}{l}0,0049 \\
0,4392\end{array}$ & $\begin{array}{l}0,2119 \\
0,4379\end{array}$ & $\begin{array}{l}0,0944 \\
0,4004\end{array}$ & $\begin{array}{l}0,3233 \\
0,2195\end{array}$ & $\begin{array}{l}0,2323 \\
0,2954\end{array}$ & $\begin{array}{l}0,1551 \\
0,6399\end{array}$ & $\begin{array}{l}\mathbf{0 , 5 2 7 4} \\
0,0575\end{array}$ \\
\hline GOSTO SALGADO & $\begin{array}{l}0,0001 \\
0,8454\end{array}$ & $\begin{array}{l}0,0970 \\
0,4936\end{array}$ & $\begin{array}{l}0,0001 \\
0,4922\end{array}$ & $\begin{array}{l}\mathbf{0 , 7 6 0 2} \\
0,3849\end{array}$ & $\begin{array}{l}0,1029 \\
0,1258\end{array}$ & $\begin{array}{l}0,0001 \\
0,9436\end{array}$ & $\begin{array}{l}0,0427 \\
0,3511\end{array}$ & $\begin{array}{l}\mathbf{0 , 9 9 5 5} \\
0,1510\end{array}$ \\
\hline GOSTO DOCE & $\begin{array}{l}0,0534 \\
0,0784\end{array}$ & $\begin{array}{l}0,2075 \\
0,5912\end{array}$ & $\begin{array}{l}\mathbf{0 , 9 7 4 2} \\
0,6189\end{array}$ & $\begin{array}{l}\mathbf{0 , 7 7 5 4} \\
0,3818\end{array}$ & $\begin{array}{l}0,0685 \\
0,7048\end{array}$ & $\begin{array}{r}\mathbf{0 , 5 6 6 8} \\
0,8469\end{array}$ & $\begin{array}{l}0,0082 \\
0,6733\end{array}$ & $\begin{array}{c}\mathbf{0 , 5 0 2 1} \\
0,3111\end{array}$ \\
\hline GOSTO ÁCIDO & $\begin{array}{l}0,0003 \\
0,4714\end{array}$ & $\begin{array}{r}\mathbf{0 , 7 2 5 8} \\
0,5059\end{array}$ & $\begin{array}{l}0,0001 \\
0,1898\end{array}$ & $\begin{array}{l}0,0032 \\
0,0744\end{array}$ & $\begin{array}{l}0,4275 \\
0,2310\end{array}$ & $\begin{array}{l}0,0001 \\
0,4908\end{array}$ & $\begin{array}{l}0,0661 \\
0,5914\end{array}$ & $\begin{array}{l}0,4212 \\
0,8357\end{array}$ \\
\hline GOSTO AMARGO & $\begin{array}{l}0,0002 \\
0,6080\end{array}$ & $\begin{array}{l}0,0031 \\
0,7566\end{array}$ & $\begin{array}{l}0,0001 \\
0,3408\end{array}$ & $\begin{array}{l}0,0328 \\
0,0644\end{array}$ & $\begin{array}{l}\mathbf{0 , 9 9 3 8} \\
0,6222\end{array}$ & $\begin{array}{l}0,0001 \\
0,5765\end{array}$ & $\begin{array}{l}0,0093 \\
0,5239\end{array}$ & $\begin{array}{l}0,0061 \\
0,1408\end{array}$ \\
\hline GOSTO UMAMI & $\begin{array}{l}0,0618 \\
0,8711\end{array}$ & $\begin{array}{l}0,1221 \\
0,5776\end{array}$ & $\begin{array}{l}0,0009 \\
0,6826\end{array}$ & $\begin{array}{l}0,0007 \\
0,4665\end{array}$ & $\begin{array}{l}0,0345 \\
0,9409\end{array}$ & $\begin{array}{l}0,0202 \\
0,4595\end{array}$ & $\begin{array}{l}0,4053 \\
0,8917\end{array}$ & $\begin{array}{l}0,1152 \\
0,2344\end{array}$ \\
\hline SABOR CARNE ASSADA & $\begin{array}{l}0,0853 \\
0,3209\end{array}$ & $\begin{array}{l}0,3096 \\
0,1750\end{array}$ & $\begin{array}{l}0,0051 \\
0,8325\end{array}$ & $\begin{array}{l}0,0017 \\
0,3948\end{array}$ & $\begin{array}{l}\mathbf{0 , 6 7 8 2} \\
0,9001\end{array}$ & $\begin{array}{l}\mathbf{0 , 9 9 9 1} \\
0,4019\end{array}$ & $\begin{array}{l}0,0001 \\
0,6604\end{array}$ & $\begin{array}{l}0,0001 \\
0,5946\end{array}$ \\
\hline SABOR CARNE FRANGO & $\begin{array}{l}0,0814 \\
0,5050\end{array}$ & $\begin{array}{l}0,0539 \\
0,2535\end{array}$ & $\begin{array}{l}0,0013 \\
\mathbf{0 , 0 2 1 3}\end{array}$ & $\begin{array}{l}0,0040 \\
0,9439\end{array}$ & $\begin{array}{l}0,2282 \\
0,4944\end{array}$ & $\begin{array}{l}0,0002 \\
0,9148\end{array}$ & $\begin{array}{l}0,2005 \\
0,3991\end{array}$ & $\begin{array}{l}0,0759 \\
0,2213\end{array}$ \\
\hline $\begin{array}{l}\text { SABOR ÓLEO VEGETAL } \\
\text { OXIDADO }\end{array}$ & $\begin{array}{l}0,0001 \\
0,8226\end{array}$ & $\begin{array}{l}0,0001 \\
0,3319\end{array}$ & $\begin{array}{l}0,0572 \\
0,3750\end{array}$ & $\begin{array}{l}0,0934 \\
0,4448\end{array}$ & $\begin{array}{l}0,0029 \\
0,7618\end{array}$ & $\begin{array}{l}0,0004 \\
0,3372\end{array}$ & $\begin{array}{l}0,0015 \\
0,3723\end{array}$ & $\begin{array}{l}0,0447 \\
0,3454\end{array}$ \\
\hline SABOR NOZ & $\begin{array}{l}0,0006 \\
0,7249\end{array}$ & $\begin{array}{l}0,0187 \\
0,1997\end{array}$ & $\begin{array}{l}0,0003 \\
0,9715\end{array}$ & $\begin{array}{l}0,0001 \\
0,3042\end{array}$ & $\begin{array}{l}0,1697 \\
0,2372\end{array}$ & $\begin{array}{l}0,2480 \\
0,3206\end{array}$ & $\begin{array}{l}0,3776 \\
0,2785\end{array}$ & $\begin{array}{l}0,2492 \\
0,4845\end{array}$ \\
\hline AFTERTASTE UMAMI & $\begin{array}{l}0,0002 \\
0,8883\end{array}$ & $\begin{array}{l}0,1267 \\
0,7979\end{array}$ & $\begin{array}{l}0,4410 \\
0,8009\end{array}$ & $\begin{array}{l}0,0198 \\
0,3753\end{array}$ & $\begin{array}{l}0,2420 \\
0,9892\end{array}$ & $\begin{array}{l}0,0008 \\
0,3642\end{array}$ & $\begin{array}{l}0,2090 \\
0,9847\end{array}$ & $\begin{array}{l}\mathbf{0 , 7 2 3 4} \\
0,1393\end{array}$ \\
\hline
\end{tabular}

Os valores indesejáveis estão marcados em negrito.

As tabelas $2 \mathrm{a}, 2 \mathrm{~b}$ e $2 \mathrm{c}$ mostram os valores de $\mathrm{F}$ calculados para amostra, provador, interação provador $\mathrm{x}$ amostra e $\mathrm{F}$ ajustado (testado contra o quadrado médio da interação). Observa-se principalmente nestas tabelas que os valores de $\mathrm{F}$ calculados para amostra e provador foram significativos $(p<0,05)$ em sua maioria. $O F$ da amostra significativo $(p<0,05)$ indica que os provadores identificaram diferenças entre pelo menos duas das amostras testadas, o que é desejável já que há discriminação das amostras. Os $\mathrm{F}$ amostra não significativos encontrados são provavelmente devidos à maior intensidade de alguns atributos que se sobressaiam mascarando os demais.

O F do provador, também significativo, indica que os provadores utilizaram diferentes porções da escala para avaliar a intensidade dos atributos, o que não é desejável, mas não é raro e é difícil de ser evitado na análise sensorial.
Por fim, os valores $\mathrm{F}$ da interação provador $\mathrm{x}$ amostra foram significativos $(p<0,05)$ em sua quase totalidade, o que pode indicar problemas no treinamento e seleção dos provadores, já que na equipe sensorial pode haver um ou mais provadores avaliando as amostras de forma não consensual com a equipe. No entanto, quando se fez o cálculo do $\mathrm{F}$ ajustado observaram-se poucos valores significativos.

Os atributos Aroma de Carne Assada e Sabor de Carne Assada apresentaram valores significativos $(\mathrm{p}<0,05)$ para o $\mathrm{F}$ interação nos três músculos e apenas no peixinho o $F$ ajustado não foi significativo. Esses atributos sofreram maior influência da experiência prévia, não sendo possível evitar durante o treinamento que houvesse a utilização da escala de forma diferente entre os provadores, mas houve consenso em relação à perda de sabor e aroma durante o armazenamento da carne assada. 
Tabela 2a -Valores de $\mathrm{F}$ amostra, $\mathrm{F}$ provador, $\mathrm{F}$ interação e $\mathrm{F}$ ajustado com a significância correspondente para avaliação de contra-filé assado

\begin{tabular}{ccccccccc}
\hline ATRIBUTO & F amostra & PR>F & F provador & PR>F & F interação & PR>F & F ajustado & PR>F \\
\hline AROMA CARNE ASSADA & 78,54 & 0.0001 & 13,77 & 0.0001 & 1.89 & 0.0268 & 41,65 & 0,0001 \\
AROMA CARNE FRANGO & 7,07 & 0.0012 & 30,35 & 0.0001 & 5,71 & 0.0001 & 1,24 & 0,3159 \\
AROMA PEIXE & 0,90 & 0.4086 & 22,86 & 0.0001 & 4,35 & 0.0001 & 0,21 & 0,8151 \\
AROMA ÓLEO LINHAÇA & 5,42 & 0.0055 & 46,49 & 0.0001 & 6,41 & 0.0001 & 0,84 & 0,4481 \\
AROMA ÓLEO VEGETAL & 0,55 & 0.5808 & 20,6 & 0.0001 & 3,01 & 0.0003 & 0,18 & 0,8361 \\
OXIDADO & 0,26 & 0.7677 & 11,79 & 0.0001 & 2,52 & 0.0021 & 0,11 & 0,9008 \\
AROMA NOZ & 8,72 & 0.0003 & 41,37 & 0.0001 & 3,83 & 0.0001 & 2,28 & 0,1346 \\
AROMA OVO COZIDO & 1.02 & 0.3633 & 12,15 & 0.0001 & 1,02 & 0.4350 & 1,00 & 0,3912 \\
AROMA PAPELÃO & 1,15 & 0.3211 & 78,73 & 0.0001 & 1,51 & 0.1044 & 0,76 & 0,4846 \\
GOSTO SALGADO & 10,22 & 0.0001 & 15,33 & 0.0001 & 3,38 & 0.0001 & 3,03 & 0,0768 \\
GOSTO DOCE & 4,66 & 0.0111 & 20,41 & 0.0001 & 3,56 & 0.0001 & 1,31 & 0,2975 \\
GOSTO ÁCIDO & 9,80 & 0.0001 & 4,14 & 0.0002 & 4,39 & 0,0001 & 2,28 & 0,1349 \\
GOSTO AMARGO & 14,74 & 0.0001 & 23,14 & 0.0001 & 2,05 & 0,0139 & 7,18 & 0,0060 \\
GOSTO UMAMI & 86,69 & 0.0001 & 19,76 & 0.0001 & 7,33 & 0.0001 & 11,82 & 0,0007 \\
SABOR CARNE ASSADA & 5,02 & 0.0079 & 15,44 & 0.0001 & 5,51 & 0,0001 & 0,91 & 0,4223 \\
SABOR CARNE FRANGO & 7,40 & 0.0009 & 12,96 & 0.0001 & 2,30 & 0.0052 & 3,22 & 0,0669 \\
SABOR ÓLEO VEGETAL & 1,25 & 0.2888 & 15,89 & 0.0001 & 3,34 & 0.0001 & 0,38 & 0,6931 \\
OXIDADO & 18,30 & 0.0001 & 22,40 & 0.0001 & 2,30 & 0.0051 & 7,95 & 0,0040 \\
SABOR NOZ & & & & & & & & \\
AFTERTASTE UMAMI & &
\end{tabular}

Tabela $2 \mathrm{~b}$-Valores de $\mathrm{F}$ amostra, $\mathrm{F}$ provador, $\mathrm{F}$ interação e $\mathrm{F}$ ajustado com a significância correspondente para avaliação de lagarto assado

\begin{tabular}{ccccccccc}
\hline ATRIBUTO & F amostra & PR>F & F provador & PR>F & F interação & PR>F & F ajustado & PR>F \\
\hline AROMA CARNE ASSADA & 54,52 & 0.0001 & 14,12 & 0.0001 & 3,54 & 0.0001 & 15,39 & 0,0002 \\
AROMA CARNE FRANGO & 16,26 & 0.0001 & 37,36 & 0.0001 & 8,98 & 0.0001 & 1,81 & 0,1956 \\
AROMA PEIXE & 8,36 & 0.0004 & 96,49 & 0.0001 & 8,97 & 0.0001 & 0,93 & 0,4144 \\
AROMA ÓLEO LINHAÇA & 13,49 & 0.0001 & 18,79 & 0.0001 & 13,00 & 0.0001 & 1,04 & 0,3769 \\
AROMA ÓLEO VEGETAL & 10,46 & 0.0001 & 36,96 & 0.0001 & 5,74 & 0.0001 & 1,82 & 0,1937 \\
OXIDADO & 10,20 & 0.0001 & 54,49 & 0.0001 & 7,89 & 0.0001 & 1,29 & 0,3016 \\
AROMA NOZ & 127,20 & 0.0001 & 121,94 & 0.0001 & 16,51 & 0.0001 & 7,71 & 0,0045 \\
AROMA OVO COZIDO & 13,71 & 0.0001 & 28,82 & 0.0001 & 12,52 & 0.0001 & 1,09 & 0,3583 \\
AROMA PAPELÃO & 6,92 & 0.0014 & 149,44 & 0.0001 & 2,66 & 0.0011 & 2,60 & 0,1051 \\
GOSTO SALGADO & 17,33 & 0.0001 & 10,46 & 0.0001 & 3,24 & 0.0001 & 5,34 & 0,0167 \\
GOSTO DOCE & 22,24 & 0.0001 & 45,98 & 0.0001 & 8,00 & 0.0001 & 2,78 & 0,0921 \\
GOSTO ÁCIDO & 2,56 & 0.0001 & 25,85 & 0.0001 & 7,62 & 0.0001 & 0,34 & 0,7199 \\
GOSTO AMARGO & 6,41 & 0.0022 & 39,56 & 0.0001 & 5,59 & 0.0001 & 1,15 & 0,3426 \\
GOSTO UMAMI & 37,95 & 0.0001 & 22,09 & 0.0001 & 2,32 & 0.0047 & 16,35 & 0,0001 \\
SABOR CARNE ASSADA & 10,19 & 0.0001 & 39,75 & 0.0001 & 9,73 & 0.0001 & 1,05 & 0,3740 \\
SABOR CARNE FRANGO & 6,97 & 0.0013 & 19,82 & 0.0001 & 4,74 & 0.0001 & 1,47 & 0,2590 \\
SABOR ÓLEO VEGETAL & 2,72 & 0.0697 & 17,41 & 0.0001 & 2,08 & 0.0126 & 1,31 & 0,2979 \\
OXIDADO & 3,31 & 0.0397 & 43,05 & 0.0001 & 5,99 & 0.0001 & 0,55 & 0,5863 \\
SABOR NOZ & & & & & & &
\end{tabular}


Os músculos têm características diferentes, por isso já se esperava que tivessem avaliações também diferentes. O peixinho teve o dobro de interações significativas $(\mathrm{p}<0,05)$, quando observado o F ajustado, em relação aos outros dois músculos. Isso poderia ser explicado pela diferença entre os músculos, mas provavelmente seja devido também ao desgaste dos provadores, já que as avaliações foram feitas em sequência e com um intervalo relativamente pequeno entre elas.

Os provadores tiveram certa dificuldade com a escala e intensidade das diferenças entre as amostras para os atributos Gosto Umami e Aftertaste Umami no contra-filé, mas não nos outros músculos. Já para os atributos Aroma de Ovo Cozido e Gosto Doce, as mesmas dificuldades foram observadas no lagarto e no peixinho, mas não no contra-filé. Essas dificuldades se devem às características diferentes de cada músculo aliadas à complexidade da matriz, mas em todos os músculos os provadores conseguiram observar de forma consensual que com o decorrer do armazenamento da carne assada os atributos não oxidativos como Aroma e Sabor de Carne Assada, Gosto umami e Aftertaste Umami, diminuíam enquanto os atributos oxidativos como Aroma e Sabor de Óleo Vegetal Oxidado, Aroma de Ovo Cozido, Aroma de Peixe e Gosto Ácido aumentavam.

Tabela 2c -Valores de F amostra, F provador, F interação e F ajustado com a significância correspondente para avaliação de peixinho assado

\begin{tabular}{ccccccccc}
\hline Atributo & F amostra & PR>F & F provador & PR>F & F interação & PR>F & F ajustado & PR>F \\
\hline AROMA CARNE ASSADA & 19,99 & 0.0001 & 16,76 & 0.0001 & 3,79 & 0.0001 & 5,28 & 0,0196 \\
AROMA CARNE FRANGO & 2,26 & 0.1087 & 24,38 & 0.0001 & 3,98 & 0.0001 & 0,57 & 0,5791 \\
AROMA PEIXE & 29,30 & 0.0001 & 164,52 & 0.0001 & 10,97 & 0.0001 & 2,67 & 0,1040 \\
AROMA ÓLEO LINHAÇA & 3,56 & 0.0316 & 20,14 & 0.0001 & 5,53 & 0.0001 & 0,64 & 0,5404 \\
AROMA ÓLEO VEGETAL & 27,73 & 0.0001 & 15,74 & 0.0001 & 3,61 & 0.0001 & 7,69 & 0,0056 \\
OXIDADO & 17,22 & 0.0001 & 85,64 & 0.0001 & 14,89 & 0.0001 & 1,16 & 0,3429 \\
AROMA NOZ & 25,62 & 0.0001 & 31,66 & 0.0001 & 4,87 & 0.0001 & 5,26 & 0,0198 \\
AROMA OVO COZIDO & 0,76 & 0.4719 & 27,38 & 0.0001 & 4,87 & 0.0001 & 0,16 & 0,8576 \\
AROMA PAPELÃO & 10,24 & 0.0001 & 51,88 & 0.0001 & 3,30 & 0.0002 & 3,11 & 0,0764 \\
GOSTO SALGADO & 9,28 & 0.0002 & 4,08 & 0.0005 & 1,88 & 0.0349 & 4,93 & 0,0239 \\
GOSTO DOCE & 22,83 & 0.0001 & 19,20 & 0.0001 & 5,90 & 0.0001 & 3,87 & 0,0460 \\
GOSTO ÁCIDO & 15,20 & 0.0001 & 8,93 & 0.0001 & 8,10 & 0.0001 & 1,86 & 0,1896 \\
GOSTO AMARGO & 4,07 & 0.0194 & 13,33 & 0.0001 & 3,76 & 0.0001 & 1,08 & 0,3656 \\
GOSTO UMAMI & 21,71 & 0.0001 & 44,07 & 0.0001 & 6,39 & 0,0001 & 3,40 & 0,0627 \\
SABOR CARNE ASSADA & 3,08 & 0.0495 & 25,24 & 0.0001 & 3,45 & 0.0001 & 0,89 & 0,4313 \\
SABOR CARNE FRANGO & 58,11 & 0.0001 & 29,64 & 0.0001 & 6,28 & 0.0001 & 9,25 & 0,0028 \\
SABOR ÓLEO VEGETAL & 20,98 & 0.0001 & 31,53 & 0.0001 & 5,43 & 0.0001 & 3,86 & 0,0461 \\
OXIDADO & 1,70 & 0.1867 & 14,81 & 0.0001 & 4,68 & 0,0001 & 0,36 & 0,7016 \\
SABOR NOZ & & & & & & &
\end{tabular}

\section{CONCLUSÕES}

A terminologia descritiva desenvolvida pode ser usada para avaliar tratamentos que visem à diminuição ou retardamento do WOF em carne assada. Os músculos analisados obtiveram avaliações diferentes, o que demonstra a necessidade do treinamento dos provadores para cada músculo a ser estudado. Atributos que os provadores considerassem bem definidos pela experiência prévia, como Aroma e Sabor de Carne Assada, provocaram dificuldades na aceitação das referências, gerando a utilização da escala de forma diferente. A maior intensidade de alguns atributos provocou maior variação na avaliação dos atributos menos intensos.

\section{REFERÊNCIAS}

1. Resconi VC, Escudero A, Campo MM. The development of aromas in ruminant meat. Molecules. 2013; 18(1): 67486781. 
2. Luciano G, Monahan FJ, Vasta V. Lipid and colour stability of meat from lambs fed fresh herbage or concentrate. Meat Science. 2009; 82(1): 193-199.

3. Tims MJ, Watts BM. Protection of cooked meats with phosphates. Food Technology. 1958; 12(1): 240-243.

4. Souza MAA, Visentainer JV, Carvalho RH, Garcia, F, Ida, EI, Shimokomaki, M. Lipid and Protein Oxidation in Charqui Meat and Jerked Beef. Brazilian Archives of Biology and Technology. 2013; 56(1): 107-112.

5. Huang WH, Greene BE. Effect of cooking method on TBA values of stored beef. Journal of Food Science. 1978. 43(1): 1201.

6. Broncano JM, Petrón MJ, Parra, V. Effect of different cooking methods on lipid oxidation and formation of free cholesterol oxidation products (COPs) in Latissimus dorsi muscle of Iberian pigs. Meat Science. 2009; 83(1): 431437.

7. Byrne DV, Bak LS, Bredie, WLP. Development of a sensory vocabulary for warmed-over flavor: Part I. In porcine meat. Journal of Sensory Studies. 1999a; 14(1): 4765.
8. Byrne DV, Bredie WLP, Martens M. Development of a sensory vocabulary for warmed-over flavor: Part II. In chicken meat. Journal of Sensory Studies. 1999b; 14(1): 67-78.

9. Byrne DV, O’Sullivan MG, Dijksterhuis GB. Sensory panel consistency during development of a vocabulary for warmed-over flavour. Food Quality and Preference. 2001; 12(1): 171-187.

10. Rossi R, Pastorelli G, Cannata, S, Tavaniello, S, Maiorano, G, Corino, C. Effect of long term dietary supplementation with plant extract on carcass characteristics meat quality and oxidative stability in pork. Meat Science. 2013; 95(1): 542-548.

11. SAS Institute Inc. Cary, USA: SAS Institute Inc; 2009. Version 9.2.

12. Civille GV, Oftedal KN. Sensory evaluation techniques - Make "good for you" taste "good". Physiology \& Behavior. 2012; 107(1):598-605.

13. Gomes CL, Pflanzer SB, Cruz AG, de Felício PE, Bolini HMA. Sensory descriptive profiling and consumer preferences of beef strip Food Research International. 2014; 59(1): 76-84. 